\title{
Flawless Identification of Fusarium Oxysporum in Tomato Plant Leaves by Machine Learning Algorithm
}

\author{
Dr. R. Dhaya, \\ Department of Computer Science, \\ Sarat Abida Campus -King Khalid University, \\ KSA, \\ dhayave12005@gmail.com
}

\begin{abstract}
In the olden days, plant diseases could be measured by visual observation and based on the level and severity of the symptoms on plant leaves. Over the day, it became a high-level degree of complexity due to the huge volume of cultivated plants. Now a day, the diseases are very different due to diverted manure procedures, and its diagnosis will be very tough even experienced farmers and agronomists too. Even though, after diagnosis, there is a lack of perfect remedy or mistaken treatment for that. The plants are affecting by many vascular fungal diseases which are widespread in many crops. Fusarium wilt (FW) is one of the fungal diseases in many plants. Mostly the tomato, sweet potatoes, tobacco, legumes, cucurbits plants are affected by this Fusarium oxysporum (FO) disease often due to its soil. The main goal of this research article is used to determine FO disease in the tomato plant leaves. Besides, the proposed algorithm constructs model with two times classifying and identifying the disease for better accuracy. The open database consists of $87 \mathrm{k}$ images with $60 \%$ affected leaves images, $40 \%$ healthy plant leaves too. Our proposed hybrid algorithm is found the disease with $96 \%$ accuracy with the huge amount of dataset.
\end{abstract}

Keywords: Plant diseases, Fusarium Oxysporum, Machine learning

\section{INTRODUCTION}

In the world, the food safety is challenging task and it is measured by many number of factors that includes plant diseases [1]. In many developing countries, the production of agricultural is coming from small holder farmers. Due to taking care of their plant by different or diverted manure procedure, monsoon change reports the loss, and more than 50\% loss due to pests and diseases by UN Environment Program (UNEP) 2013 annual report. In earlier days, the plant diseases could be measured by visual observation and based on the level and severity of the symptoms on plant leaves. Over the course of the day, it became high level degree complexity due to huge volume of cultivated plants. Now a day, the diseases are very different due to diverted manure procedure and its diagnosis will be very tough even experienced farmer and agronomist too. Even though, after diagnosis, there is an absence of perfect remedy or mistaken treatment for that. Figure 1 shows FO disease in tomato plant leaves.

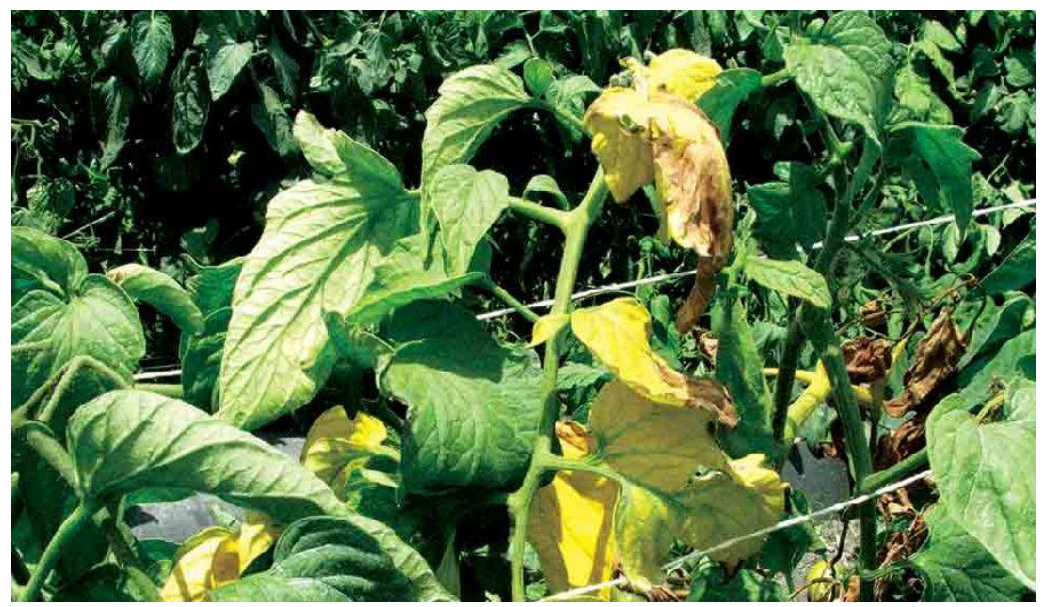

Figure 1 Fusarium Oxysporum disease in tomato plant leaves

ISSN: 2582-4252 (online)

Submitted: 05.12.2020

Revised: 12.01 .2021

Accepted: 03.02.2021

Published: 17.02.2021 
Journal of Innovative Image Processing (JIIP) 2020)

Vol.02/ No. 04

Pages: 194-201

https://www.irojournals.com/iroiip/

DOI: https://doi.org/10.36548/jiip.2020.4.004

Also Piper betle $\mathrm{L}$. $(\mathrm{PbC})$ is used to control FW of tomato leaves caused by FO with the help of alteration of carbendazin [2]. It was the primeval technique is to rectify the disease appears in the plant. Based on shoot, root growth, of any plant seedlings can be control in healthy way with $\mathrm{PbC}$ treated plants or spinach leaves [3]. Also the electron microscopy training is used to acquire the infestation in the plant vascular bundles. In addition, it is used to show the combining of total phenolics from the root tissue $[4,5,6]$. Figure 2 shows effects of FO disease.

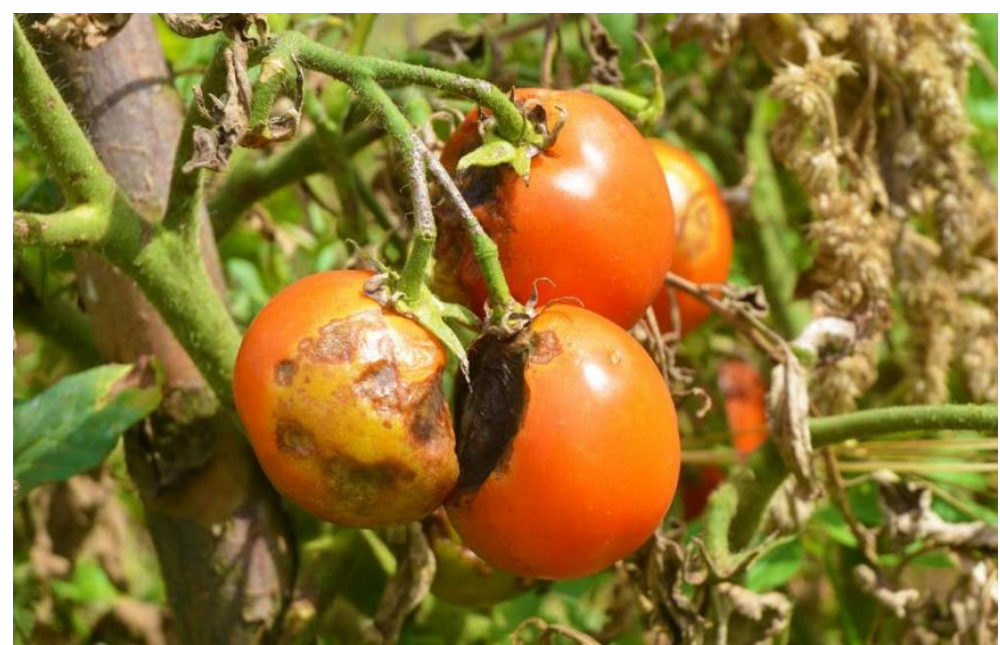

Figure 2 Effects of Fusarium Oxysporum disease in tomato plant

This research article is focusing diseases in tomato plant especially affected by FO in common. Recently, the image processing is used to rectify the diseases occurring in tomato leaves by different type of image processing and recognition based algorithms $[7,8]$. The figure 3 shows the types of tomato plant leaves for classification for improved accuracy in the prediction.

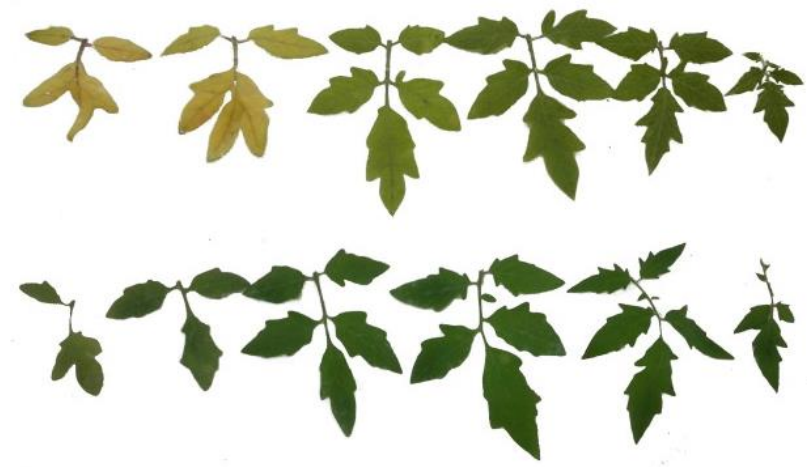

Figure 3 Types of tomato plant leaves for classification

\section{ORGANIZATION OF THE RESEARCH}

The structure of the research article organized as follows; Section 3 gives literature survey of recent prediction of FO disease in various plants. Section 4 provides the description of methodology. Section 5 delivers description of results and discussion, finally the conclusion and further improvement is in section 6.

\section{PRELIMINARIES}

The agronomist is predicting disease and diagnosis via manual presence and from observation of plant leaves. The automated computation system is designed and performed by mohanty et al [9]. Also it is an example and pioneer of simple mobile application which is very easy accessible device for modernize formers. LeCun et al develops Graphics Processing Unit (GPU) processor for predicting diseases in plants by Machine learning 
Journal of Innovative Image Processing (JIIP) 2020)

Vol.02/ No. 04

Pages: 194-201

https://www.irojournals.com/iroiip/

DOI: https://doi.org/10.36548/jiip.2020.4.004

algorithm. And they discussed novel methods and models for finding the diseases in plants. He believes in image recognition and deep learning for two sector identification. Also the voice recognition for the complex tasks with huge amount of dataset gives effective prediction [10]. Carranza-Rojas et al introduces deep learning techniques for finding the disease in plant [11]. Yang and Guo propose the detection methods for plant diseases. The powerful technique Convolutional Neural Network (CNN) in deep learning tool introduces to detect disease in plant. The deep learning algorithm can be processed huge amount of data and gives better accuracy [12]. Lee et al proposes CNN methods to recognize the plant based on its leaves images [13]. Grinblat et al construct the neural network for the perfect recognizing the various different species with the help of its leaves [14].

\section{RESEARCH GAP}

Many research articles go pear-shaped in predicting FO disease in plant and erroneous results built by them. Also many research articles are saying as a future work that prediction by best classifier or combination of classifiers like as hybrid. Our proposing techniques consist of combined image processing and classifier algorithm. It can provide better accurate results in prediction of FO disease in plant's leaves.

\section{METHODOLOGIES}

In our project, many successive stages are used to identify the FO diseases in tomato leaves. The real raw images are noisy and unclear vision. So there is performing preprocessing by our proposed hybrid algorithm for removal of noise and clear images. Then the image classification and recognition takes place after that $[15,16]$. Generally, FO disease in the leaves consist of yellowing of older leaves and stem of the plant will be discoloration. Also some black patches may be present on the roots. After a long time, the fungus can form and survive on seeds too.

\section{$\underline{\text { Successive stages }}$}

\subsection{Initial Stage Prediction}

In our proposed methodology, we introduce two-factor identification based on two types of datasets for classification for accurate results. Initially, the identification of disease is done by image processing method with less amount of data set of an overall picture of plants leaves. After image registration, the tomato plant leaves will be checked by an image recognition algorithm. Based on plant and leaves condition algorithm can be classified disease affected or healthy one. Even though healthy plant condition, algorithm advising it to second stage deep classification. But if it is found as a disease in a plant; alarm can be activated which is shown in figure 4.

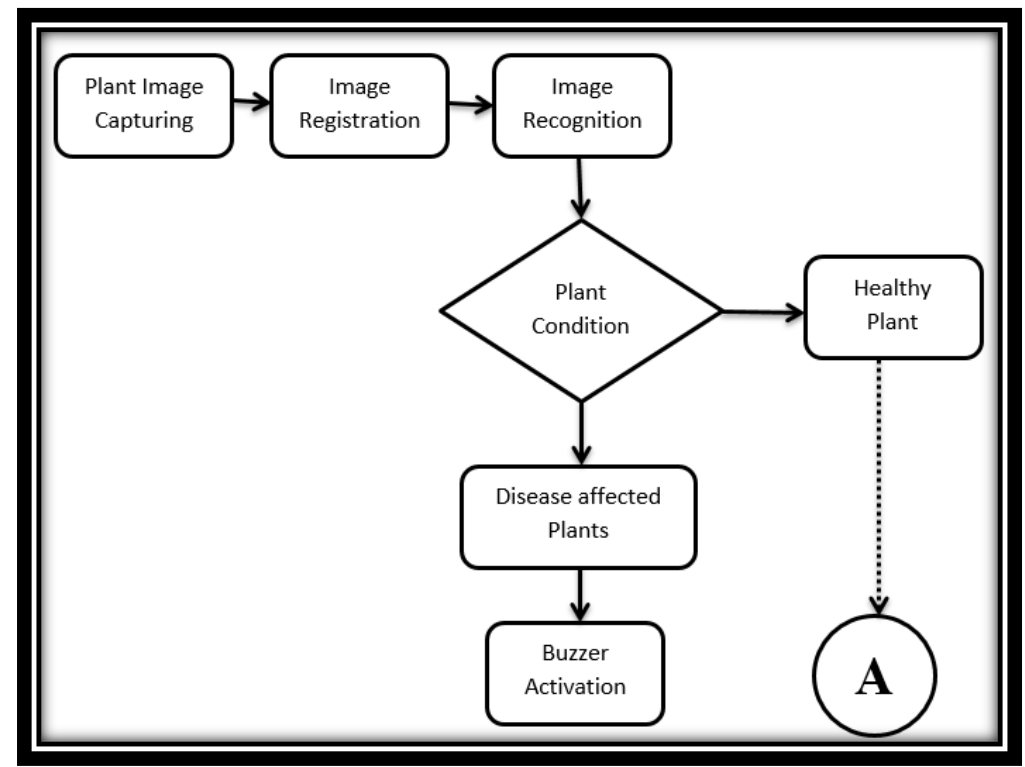

Figure 4 Prediction at early stage

ISSN: 2582-4252 (online)

Submitted: 05.12.2020

Revised: 12.01 .2021

Accepted: 03.02.2021

Published: 17.02.2021 
Journal of Innovative Image Processing (JIIP) 2020)

Vol.02/ No. 04

Pages: 194-201

https://www.irojournals.com/iroiip/

DOI: https://doi.org/10.36548/jiip.2020.4.004

Figure 5 shows the healthy plant identification by image recognition method in image processing.
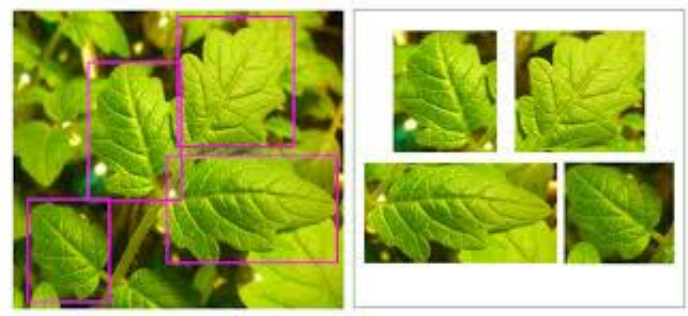

Figure 5 Healthy plant identification by Image Recognition

\subsection{VTT Phase}

Many erroneous predictions happened even after the image recognition method. So our proposed methodology tries to solve this problem with aid of the VTT phase. This phase comprises validation, training, and testing of data for better prediction. Therefore, from the first stage, the healthy plant images will be preprocessing for the machine learning algorithm. This image will be classifying features in the data collection section [17]. Figure 6 shows the VTT phase section in our proposed method. This section can be classified into three sections as follows;

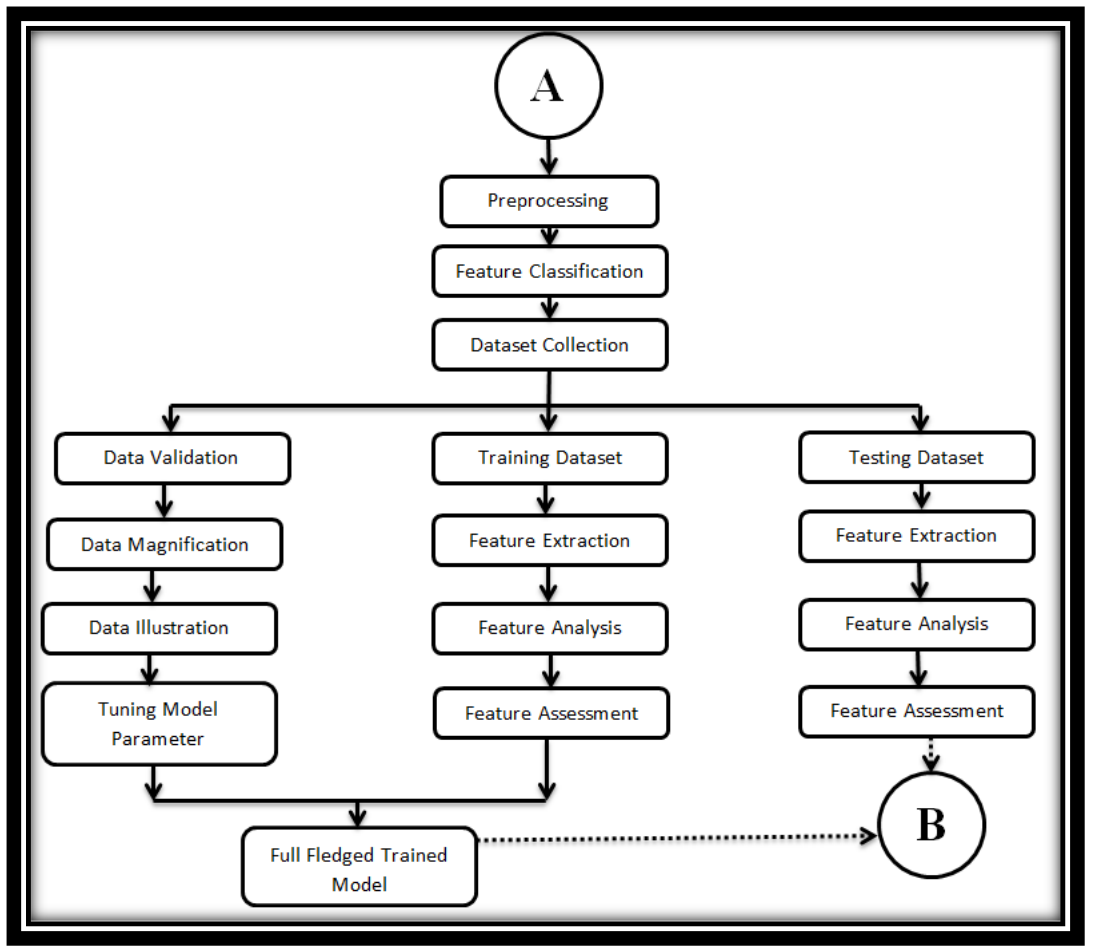

Figure 6 VTT Phase at Proposed framework

\section{Data validation}

Mainly this data validation is the process for complex constraints. This complex processing is operated within the system and having a set of processes.

\section{Data magnification}

It is the process of enlarging the tomato plant's leaves in an image. This enlarging will give better visual clarity from the tomato plant's leaves images and is called magnification.

ISSN: 2582-4252 (online)

Submitted: 05.12.2020

Revised: 12.01.2021

Accepted: 03.02.2021

Published: 17.02.2021 
Journal of Innovative Image Processing (JIIP) 2020)

Vol.02/ No. 04

Pages: 194-201

https://www.irojournals.com/iroiip/

DOI: https://doi.org/10.36548/jiip.2020.4.004

\section{Data illustration}

This is the process of data translation information for identifying patterns in a better way. For a better view of visual context, this data illustration categorizing the many patterns in the large datasets. It may help to achieve better accuracy [18]. The validation and training data model provides the full-fledged trained model and it compares with testing data of feature assessment. This feature assessment continues with the final stage prediction which is shown in figure 7.

$\mathrm{P}(\mathrm{A})=$ Prior probability of class

$$
P\left(\frac{A}{t_{l}}\right)=\frac{P\left(t_{l} / A\right) \cdot P(A)}{P\left(t_{l}\right)}
$$

$P\left(t_{l} / A\right)=$ The probability of predictor by given class

$P\left(t_{l}\right)=$ Prior probability of target predictor (Tomato Leaf)

$P\left(\frac{A}{t_{l}}\right)=$ Posterior Probability of class A given by the predictor

\subsection{Final Stage Prediction}

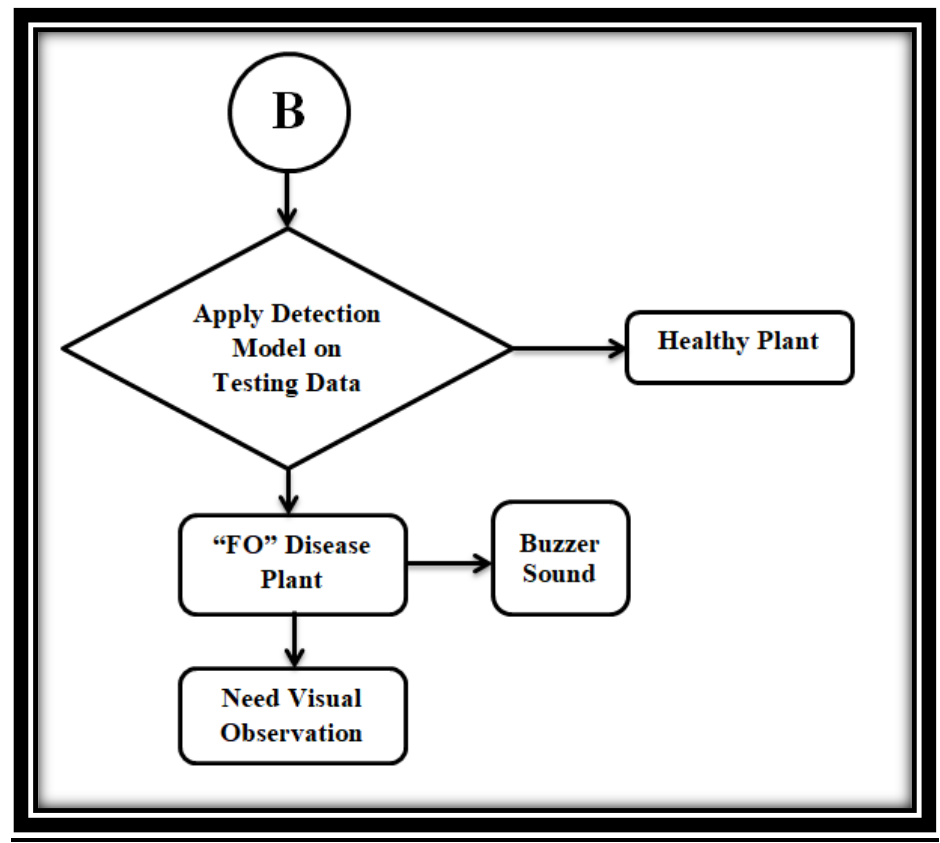

Figure 7 Detection model for FO disease in final stage

The detection model is in the final stage of prediction processing units. This can be classified and identified with the ML algorithm [19]. Again if it is found as a healthy plant gives a positive reply and no need for manual inspection. If the FO disease identifies in the leaf of a tomato, an algorithm is used to activate an alarm to need in of visual observation. The systematic structure of Naïve Bayes approach is shown in figure 8.

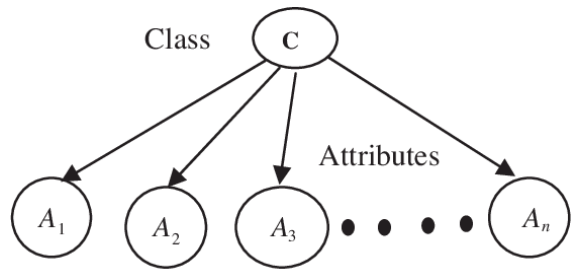

Figure 8 Structure of Naïve Bayes approach

ISSN: 2582-4252 (online)

Submitted: 05.12.2020

Revised: 12.01.2021

Accepted: 03.02.2021

Published: 17.02.2021 
Journal of Innovative Image Processing (JIIP) 2020)

Vol.02/ No. 04

Pages: 194-201

https://www.irojournals.com/iroiip/

DOI: https://doi.org/10.36548/jiip.2020.4.004

\section{RESULTS DISCUSSION}

Our proposed algorithm consists of naïve Bayes with an image recognition method. First level checking gives a moderate prediction of plant disease and second level prediction will be full-fledged. Figure 6 shows the leaf collection for image recognition. Figure 9 shows different condition of tomato leaves for classification purpose.

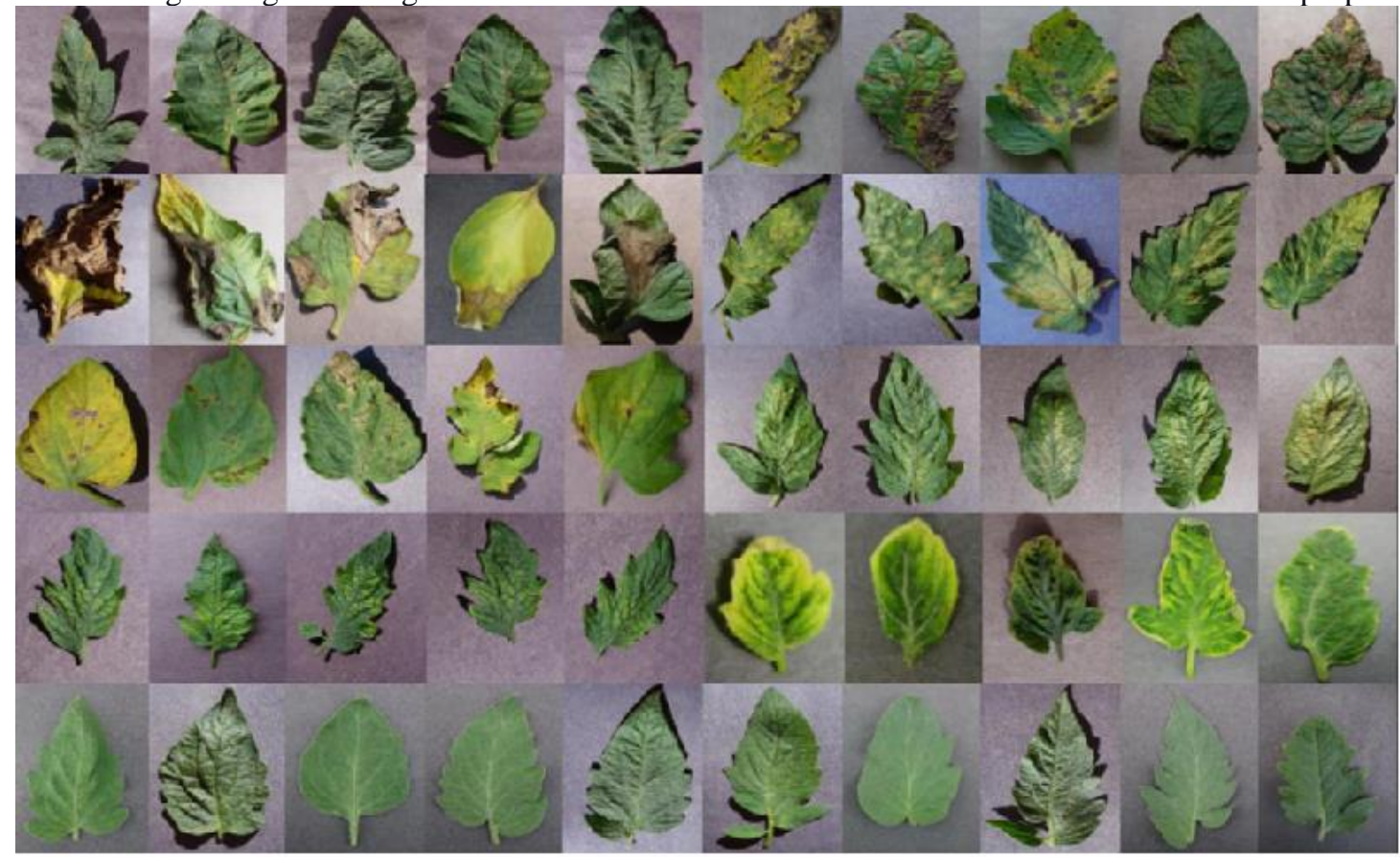

Tomato Leaves for Classification

Figure 9 Image classification for leaf

Due to soil-inhabiting, the fungus on tomato leaf is named Fusarium oxysporum. Affected leaves are hard to live and the plant gives spoiled fruits that are entirely wasted. Removing the infected plant from the area gives more effective cultivation in further tomato plants in that same area. Also, we recommend crop rotation and soil changing also provides good results against FO disease. Figure 10 shows the histogram of the tomato leaf image which is used to determine the distribution of pixels in the images.

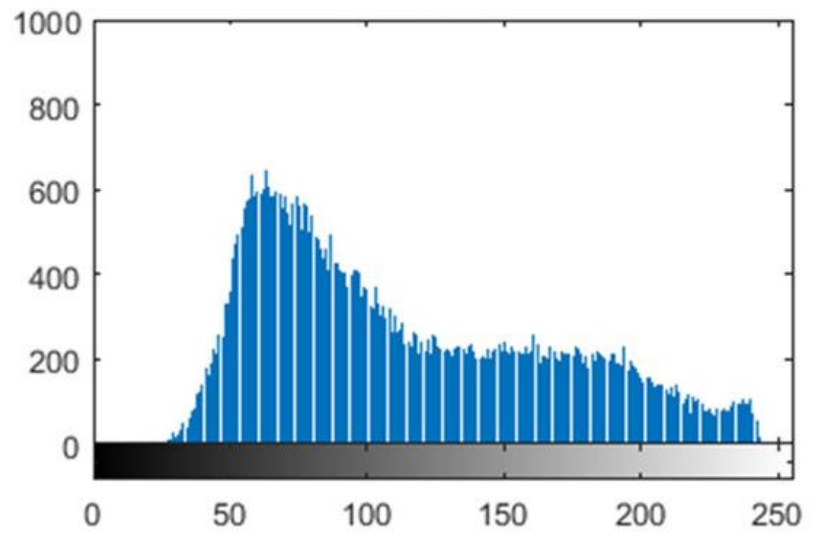

Figure 10 Histogram of leaf image

ISSN: 2582-4252 (online)

Submitted: 05.12.2020

Revised: 12.01.2021

Accepted: 03.02.2021

Published: 17.02.2021 
Journal of Innovative Image Processing (JIIP) 2020)

Vol.02/ No. 04

Pages: 194-201

https://www.irojournals.com/iroiip/

DOI: https://doi.org/10.36548/jiip.2020.4.004

Figure 11 shows the comparison chart of three different measurements of FO and its prediction accuracy rate. The image processing techniques are showing high accuracy in less number of image's data set. Also, it's cannot be performed in a huge amount of image datasets due to the very high executing time for the algorithm. Our proposed algorithm contains the higher accuracy due to two times confirmation from different sector algorithm.

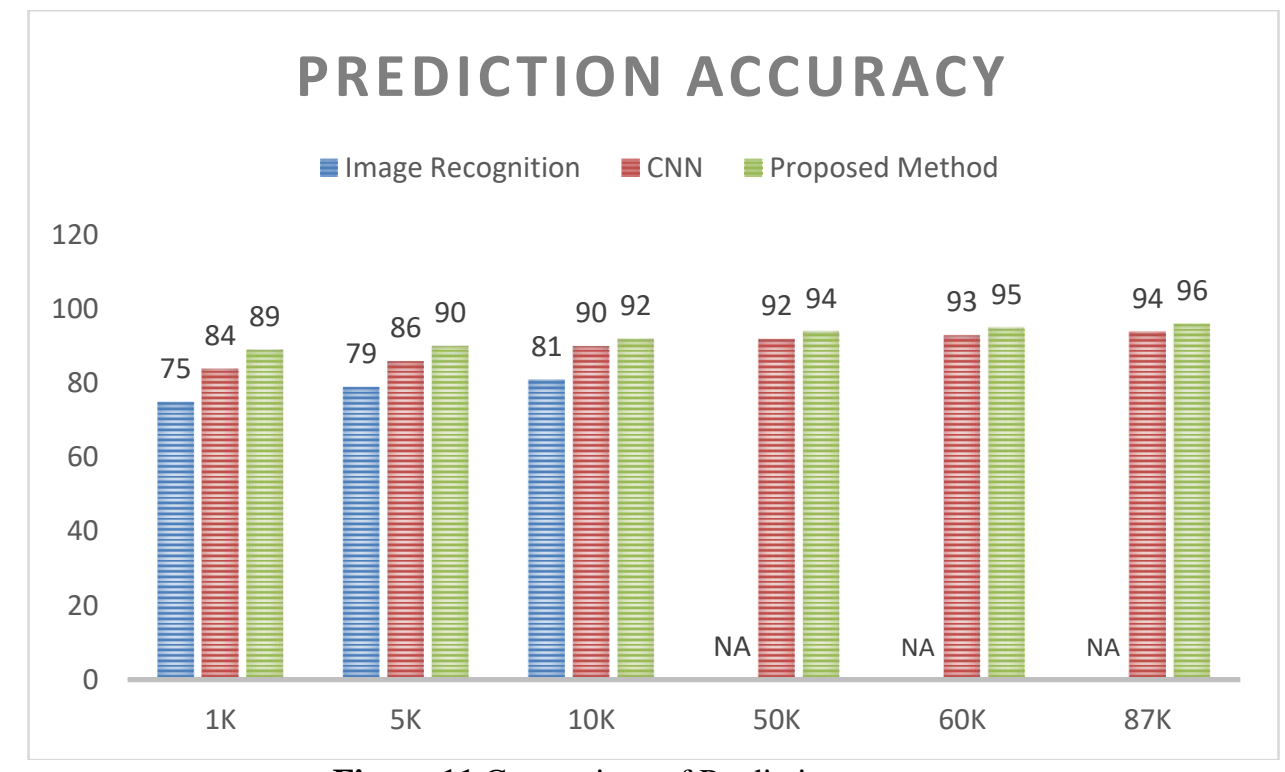

Figure 11 Comparison of Prediction accuracy

Finally, the prediction accuracy calculation after 50K image dataset will give more execution time while used image processing algorithms which shows in figure 11 as Not Available (NA).

\section{CONCLUSION}

This paper explained our proposed techniques which consist of successive phases to get better accurate results. We achieved better accurate results due to the two-factor identification method. A single factor identification method provided less accuracy even used with single-factor identification either image recognition methods or ML algorithm. We obtained good prediction accuracy of our proposed classifier method to identify the FO disease.

\section{Future tasks}

We can develop further support for early warning prediction of plant disease after making a high accuracy rate model in real-time conditions [18]. In a large number of plants site, we are planning to implement with location finding too. This will make it easier to the identification of disease plants very accurately with time consumption. Also, the images can be captured in the plant with the help of a flying drone and they can be classified in our future work. The author strongly believes this article can be useful for modernizing farmers and agronomists.

\section{REFERENCES}

[1] Strange, Richard \& Scott, Peter. (2005). Plant Disease: A Threat to Global Food Security. Annual review of phytopathology. 43. 83-116. 10.1146/annurev.phyto.43.113004.133839.

[2] S. Kouki, N. Saidi, A. Ben Rajeb, M. Brahmi, A. Bellila, M. Fumio, A. Hefiène, N. Jedidi, J. Downer, H. Ouzari, "Control of Fusarium Wilt of Tomato Caused by Fusarium oxysporum F. Sp. Radicis-Lycopersici Using Mixture of Vegetable and Posidonia oceanica Compost", Applied and Environmental Soil Science, vol. 2012, Article ID 239639, 11 pages, 2012. https://doi.org/10.1155/2012/239639

[3] Correll, James C.. (1994). Economically Important Diseases of Spinach. Plant Disease. 78. 653. 10.1094/PD-780653.

ISSN: 2582-4252 (online)

Submitted: 05.12.2020

Revised: 12.01 .2021

Accepted: 03.02.2021

Published: 17.02.2021 
Journal of Innovative Image Processing (JIIP) 2020)

Vol.02/ No. 04

Pages: 194-201

https://www.irojournals.com/iroiip/

DOI: https://doi.org/10.36548/jiip.2020.4.004

[4] Kim, Da-Ran \& Jeon, Chang-Wook \& Shin, Jae-Ho \& Weller, David \& Thomashow, Linda \& Kwak, Youn-Sig. (2018). Function and Distribution of a Lantipeptide in Strawberry Fusarium Wilt Disease-Suppressive Soils. Molecular Plant-Microbe Interactions. 32. 10.1094/MPMI-05-18-0129-R.

[5] Atila, Umit \& Ucar, Murat \& Akyol, Kemal \& Uçar, Emine. (2021). Plant leaf disease classification using EfficientNet deep learning model. Ecological Informatics. 61. 101182. 10.1016/j.ecoinf.2020.101182.

[6] M. A. Jasim and J. M. AL-Tuwaijari, "Plant Leaf Diseases Detection and Classification Using Image Processing and Deep Learning Techniques," 2020 International Conference on Computer Science and Software Engineering (CSASE), Duhok, Iraq, 2020, pp. 259-265, doi: 10.1109/CSASE48920.2020.9142097.

[7] Azlah, M.A.F.; Chua, L.S.; Rahmad, F.R.; Abdullah, F.I.; Wan Alwi, S.R. Review on Techniques for Plant Leaf Classification and Recognition. Computers 2019, 8, 77. https://doi.org/10.3390/computers8040077

[8] Pegg, Kenneth \& Coates, Lindel \& O’Neill, Wayne \& Turner, David. (2019). The Epidemiology of Fusarium Wilt of Banana. Frontiers in Plant Science. 10. 1395. 10.3389/fpls.2019.01395.

[9] Mohanty, Sharada \& Hughes, David \& Salathe, Marcel. (2016). Using Deep Learning for Image-Based Plant Disease Detection. Frontiers in Plant Science. 7. 10.3389/fpls.2016.01419.

[10] LeCun, Y., Bengio, Y., Hinton, G., 2015. Deep learning. Nature 521, 436-444. http://dx. doi.org/10.1038/nature14539.

[11] Carranza-Rojas, J., Goeau, H., Bonnet, P., Mata-Montero, E., Joly, A., 2017. Going deeper in the automated identification of Herbarium specimens. BMC Evol. Biol. http://dx. doi.org/10.1186/s12862-017-1014-z.

[12] Yang, X., Guo, T., 2017. Machine learning in plant disease research. Europ. J. BioMed. Res. 6-9. http://dx.doi.org/10.18088/ejbmr.3.1.2016.pp6-9.

[13] Lee, S.H., Chan, C.S., Wilkin, P., Remagnino, P. 2015. Deep-plant: Plant identification with convolutional neural networks. 2015 IEEE Intl Conf. on Image Processing, pp. 452-456.

[14] Grinblat, G.L., Uzal, L.C., Larese, M.G., Granitto, P.M., 2016. Deep learning for plant identification using vein morphological patterns. Comput. Electron. Agric. 127, 418-424.

[15] Omeer, Abdulla \& Deshmukh, Ratnadeep. (2021). Improving the classification of invasive plant species by using continuous wavelet analysis and feature reduction techniques. Ecological Informatics. 61. 101181. 10.1016/j.ecoinf.2020.101181.

[16] Mohanty, S.P., Hughes, D.P., Salathé, M., 2016. Using deep learning for image-based plant disease detection. Front. Plant Sci. 7http://dx.doi.org/10.3389/fpls.2016. 01419. Article: 1419.

[17] Ferentinos, Konstantinos. (2018). Deep learning models for plant disease detection and diagnosis. Computers and Electronics in Agriculture. 145. 311-318. 10.1016/j.compag.2018.01.009.

[18] Elhadi Adam, Houtao Deng, John Odindi, Elfatih M. Abdel-Rahman, Onisimo Mutanga, "Detecting the Early Stage of Phaeosphaeria Leaf Spot Infestations in Maize Crop Using In Situ Hyperspectral Data and Guided Regularized Random Algorithm", Journal ofSpectroscopy, vol. 2017, ArticleID 6961387, 8 pages, 2017. https://doi.org/10.1155/2017/6961387

[19] Shin, Jihoon \& Yoon, Seong Hyeon \& Kim, YoungWoo \& Kim, Taeho \& Go, ByeongGeon \& Cha, Yoonkyung. (2021). Effects of class imbalance on resampling and ensemble learning for improved prediction of cyanobacteria blooms. Ecological Informatics. 61. 101202. 10.1016/j.ecoinf.2020.101202.

ISSN: 2582-4252 (online)

Submitted: 05.12.2020

Revised: 12.01 .2021

Accepted: 03.02.2021

Published: 17.02.2021 